\title{
Como a Análise do Comportamento tem Contribuído para Área da Saúde?
}

\author{
Ramon Marin ${ }^{1}$ \\ Pedro Bordini Faleiros ${ }^{2}$ \\ ${ }^{1}$ Universidade Federal de São Carlos, SP, Brasil. \\ ${ }^{2}$ Universidade Metodista de Piracicaba, SP, Brasil. \\ Antonio Bento Alves de Moraes ${ }^{3}$ \\ ${ }^{3}$ Universidade de Campinas, SP, Brasil.
}

Resumo: A Psicologia da Saúde tem investigado variáveis e proposto estratégias de prevenção e promoção de problemas de saúde. Especificamente na Análise do Comportamento Aplicada o foco tem sido a alteração e manutenção de comportamentos relacionados à saúde. Por meio de uma revisão de literatura, o objetivo deste trabalho foi identificar estudos, pautados na Análise do Comportamento Aplicada, que apresentassem contribuições à área de Saúde. Para tanto, foram revisadas todas as edições do Journal of Applied Behavior Analysis, publicadas entre 2000 e 2015. Os artigos que continham em seus títulos ou resumos, termos relacionados à saúde foram selecionados. Ao todo 160 artigos foram encontrados e após análise dos mesmos, considerando, o objetivo, métodos e os resultados, 11 temas relacionados à saúde foram definidos (adesão, diabetes, problemas alimentares, uso de drogas, procedimentos hospitalares, saúde ocupacional, saúde bucal, atividade física, comportamento autolesivo, problemas de sono e segurança no trânsito). Os estudos baseados nos pressupostos analíticos comportamentais demonstram a aplicabilidade dos conceitos na área de saúde. Além disto, foi também possível identificar e analisar as principais características metodológicas utilizadas e sua eficácia para a alteração de padrões de comportamentos de saúde.

Palavras-chave: Psicologia da Saúde, Análise do Comportamento Aplicada, Revisão de Literatura.

\section{How Behavioral Analysis has Contributed to the Health Area?}

\begin{abstract}
Health Psychology has investigated variables and proposed strategies to prevent problems or promote health conditions. Specifically in Applied Behavior Analysis, the maintenance and modifications of health-related behaviors has been the focus of interest. By a literature review, the aim of this work was to identify studies, based on Applied Behavior Analysis, that show contributions to the health area. All issues of Journal of Applied Behavior Analysis published between 2000 and 2015 were reviewed. The articles that had, in their title, any healthrelated terms were selected. One hundred and sixty studies were found and after the analysis, considering the objective, methods and results, 11 health themes were defined (compliance, diabetes, feeding problems, drugs use, medical procedures, occupational health, oral health, physical activity, self-injurious behavior, sleeping problems and traffic safety). Studies based on behavior analytical proposals showed the applicability of concepts to the health area. Besides, it was possible to identify and analyze the main methodological characteristics used and also their efficacy to changing health behavior patterns.
\end{abstract}

Keywords: Health Psychology, Behavior Analysis, Literature Review. 


\title{
¿Como ha contribuido el Análisis de Comportamiento al Campo de la Área de Salud?
}

\begin{abstract}
Resumen: La Psicología de la Salud ha investigado variables y propuesto estrategias para la prevenciónypromocióndeproblemasdesalud.EspecíficamenteenelAnálisisdeComportamiento Aplicado, la atención se ha centrado en cambiar y mantener los comportamientos relacionados con la salud. A través de una revisión de la literatura, el objetivo de este trabajo fue identificar estudios, basados en el Análisis de Comportamiento Aplicado, que presentaran contribuciones al área de Salud. Para este fin, todas las ediciones del Journal of Applied Behavior Analysis, publicadas entre 2000 y 2015 En total, 160 artículos fueron encontrados y después del análisis de los mismos, considerando, el objetivo, métodos y resultados, 11 temas relacionados a la salud fueron definidos (adhesión, diabetes, problemas alimentarios, uso de drogas, procedimientos hospitalarios, salud ocupacional, salud bucodental, actividad física, comportamiento autolesivo, problemas de sueño y seguridad en el tránsito). Los estudios basados en los supuestos analíticos comportamentales demuestran la aplicabilidad de los conceptos en el área de salud. Además, fue también posible identificar y analizar las principales características metodológicas utilizadas y su eficacia para la alteración de patrones de comportamientos de salud. Se seleccionaron los artículos que contenían términos relacionados con la salud en sus títulos o resúmenes. Se encontraron un total de 160 artículos y después de analizarlos, considerando el objetivo, los métodos y los resultados, se definieron 11 temas relacionados con la salud (adherencia, diabetes, problemas dietéticos, uso de drogas, procedimientos hospitalarios, salud ocupacional, salud bucodental, actividad física, comportamiento auto-lesivo, problemas para dormir y seguridad vial). Los estudios basados en suposiciones analíticas de comportamiento demuestran la aplicabilidad de los conceptos en el campo de la salud. Además, también fue posible identificar y analizar las principales características metodológicas utilizadas y su efectividad para cambiar los patrones de comportamiento de salud.
\end{abstract}

Palabras clave: Psicología de la Salud, Análisis del Comportamiento Aplicado, Revisión de Literatura.

A Psicologia da saúde pode ser definida como o agregado de contribuições educacionais, profissionais e científicas de múltiplas abordagens para a promoção e manutenção de estados saudáveis em indivíduos (Engel, 1980; Matarazzo, 1982). No âmbito da atuação profissional, a Psicologia da Saúde tem proposto estratégias de promoção de saúde e prevenção de problemas, com base na compreensão dos múltiplos fatores existentes na relação entre o sujeito e seu ambiente (Gorayeb, 2010; Gorayeb, \& Guerrelhas, 2003; Straub, 2014; Taylor, 1990, 1999; Teixeira, 2004), especificamente na análise das funções das respostas que este emite, visando alterar ou manter estados de saúde (Mechanic, 1962; Starling, 2001).

Considerando estas múltiplas interações entre o sujeito e o ambiente, poderíamos pensar que, um dos fatores fundamentais na manutenção de estados de saúde são os padrões comportamentais de cada indivíduo. Sabe-se que as respostas emitidas pelos sujeitos implicam diretamente em seus estados de saúde (Gorayeb, 2010; Taylor, 1990). Sendo assim, uma das ciências que pode contribuir com a análise e intervenção na saúde individual ou coletiva, como propõe a Psicologia da Saúde, é a Análise do Comportamento.

Para a Análise do Comportamento, segundo Banaco (1999), Gon, Zakir, Andrade, Gavazzoni e Brandenburg (2015) e Matos (1999), uma resposta, seja ela considerada "saudável" ou não, foi selecionada pelas consequências e, portanto, é adaptativa as condições ambientais e integrada ao repertório do sujeito, gerando a necessidade de compreende-la funcionalmente para possíveis intervenções. A compreensão de quais contingências afetam ou afetaram o comportamento do sujeito é de vital importância para 
as intervenções na saúde (Kerbauy, 2002). A forma como um sujeito se comporta altera as condições integrantes do continuum saúde-doença (Gon et al., 2015; Skinner, 1991), o que configura uma complexa inter-relação entre as respostas que o sujeito emite, as variáveis que controlam seus comportamentos e, também, as múltiplas condições que promovem ou não estados de saúde (Kubo, \& Botomé, 2001). A compreensão de tais fatores, faz-se por considerar na aplicação de aspectos teóricos-experimentais em múltiplos contextos, para qual Baer, Wolf e Risley (1968) propuseram critérios que caracterizariam ou não a aplicação correta da análise do comportamento em contextos de alta relevância social.

Para compreender o modo como a Análise do Comportamento se aplica em contextos de saúde, Calheiros (2016), Calheiros, Amaral, Gon e Costa Júnior (2016), Marin, Moraes e Faleiros, (2017), Moraes e Rolim (2013), realizaram revisões de literatura, com trabalhos que, por meio de pressupostos analítico comportamentais, manipularam ou descreveram variáveis que interferem nos estados de saúde

Moraes e Rolim (2013) realizaram uma revisão em todos os 27 volumes da coleção Sobre Comportamento e Cognição, no período s de 1997 a 2010. Os autores tinham como objetivo analisar a contribuição da Análise do Comportamento para a Psicologia da Saúde e para tanto, buscaram capítulos que estivessem voltados a área. Nesta revisão, foram identificados os temas: "comportamento de risco", "tabagismo", "etilismo", "câncer", "diabetes”, "saúde mental e emocional", "ansiedade", "depressão", "modelos biomédicos e biopsicossociais", "sistemas de saúde", "enfermaria", "gestantes", "relação profissional-paciente”, "saúde bucal", "transtorno dismórfico" e "terapia ocupacional”. Dos 1.023 capítulos publicados na coleção, 88 deles (52 estudos teóricos e 36 empíricos) foram identificados pelos autores como os que se relacionaram com a área de saúde e fundamentados na análise do comportamento. Em comparação ao total de publicações existentes na coleção, os capítulos de análise do comportamento e saúde, representaram $9 \%$.

Calheiros et al. (2016) buscaram em bases de dados de acesso livre (Biblioteca Virtual de Saúde, Scientific Electronic Library Online - SciELO e Literatura Latino-Americana e do Caribe em Ciências da Saúde) artigos fundamentados na análise do comportamento e relacionados a área da Saúde, nos últimos dez anos (2005 a 2014). Foram realizadas duas buscas, a primeira utilizando palavras-chave como "análise do comportamento", "medicina do comportamento", "saúde" e "psicologia da saúde". Na segunda, foram usadas como palavras-chave "análise do comportamento” em combinações com o nome das 53 especialidades médicas consideradas pelo Conselho Nacional de Medicina. Os autores analisaram 789 artigos, dos quais, ao final das duas etapas de seleção de dados, foram selecionados apenas sete artigos $(0,88 \%)$, que revelaram uma aplicabilidade da análise do comportamento à área de saúde, embora o baixo número de artigos em evidência. Esta quantidade restrita de artigos reflete, segundo os autores, pode ter sido consequência de fatores como a falta de definições conceituais, demonstrando a falta de precisão em pesquisas da área. O formato e o foco das buscas realizadas por esta revisão, hora apontam dados relevantes à área biológicas, hora apontam dados relevantes à análise do comportamento. Neste sentido, os autores argumentam que a forma imprecisa das pesquisas pode não produzir uma interrelação entre áreas como análise do comportamento e saúde.

Em outro estudo, (Calheiros, 2016), buscou teses fundamentadas na Análise do Comportamento relacionadas a doenças crônicas não transmissíveis (DCNT), em dois bancos de dados abertos (Banco de Teses da Coordenação de Aperfeiçoamento de Pessoal de Nível Superior - CAPES e Biblioteca Digital Brasileira de Teses e Dissertações). Por meio das palavras-chave "análise do comportamento" AND "Saúde" e utilizando como critério de seleção os pressupostos da Análise do Comportamento Aplicada, de Baer et al. (1968, 1987). Foram encontradas 54 dissertações e 14 teses em ambas bases de dados. No entanto, apenas três teses atenderam a todos os critérios estabelecidos pelo procedimento de seleção. Segundo a autora, embora exista um grande número de pessoas que apresentam diagnóstico de DCNT, o número de participantes em cada estudo foi pequeno. Ainda de acordo com Calheiros (2016), características relacionadas ao uso de delineamentos de sujeito único por pesquisas em Análise do Comportamento, além de ocasionar em números menores de participantes, poderiam resultar em estilos metodológicos para melhor execução de procedimentos na pesquisa e também no estudo aplicado a área de saúde.

Marin et al. (2017) realizaram outra revisão de literatura com o objetivo de identificar os temas estudados na área de saúde, em trabalhos empíricos base- 
ados na abordagem comportamental, utilizando-se a base de dados "Periódicos CAPES". Foram encontrados 3.495 artigos, dos quais foram selecionados estudos empíricos da área de saúde embasados na análise do comportamento que tinham sido realizados com sujeitos humanos. Desse número foram selecionados 68 artigos, publicados no período de 1980 a 2014, que atenderam os critérios estabelecidos pelos pesquisadores. A partir da leitura dos resumos, os artigos foram classificados em três grandes temas, "Distúrbios", "Intervenções" e "Padrões Comportamentais". Os autores mantiveram o foco somente nos temas relacionados a saúde, não produzindo análises pautadas nos procedimentos e resultados de cada estudo.

Os autores apontaram um aumento no número de publicações a partir do início do século XXI, além de uma baixa concentração de estudos encontrados em periódicos nacionais, sendo que 52 artigos foram publicados no Journal of Applied Behavior Analysis (JABA). No entanto, Marin et al. (2017) sugerem que algumas características metodológicas podem ter restringido a busca e a análise dos artigos. Assim como apontado por Calheiros et al. (2016) os autores argumentam, por exemplo, que os termos utilizados na busca (health e behavior analysis) não foram específicos o suficiente para a busca. Portanto, palavras chaves mais significativas para a Análise do Comportamento (punishment, reinforcement, contingency etc.), ou outros métodos de busca poderiam ter produzido dados mais fidedignos com a realidade das publicações, no entanto apresentando possíveis restrições aos dados encontrados com base em Psicologia da Saúde.

A partir destas quatro revisões apresentadas (Calheiros, 2016; Calheiros et al., 2016; Marin et al., 2017; Moraes, \& Rolim, 2013), pode-se dizer que há um contínuo, e até mesmo possível crescente, interesse da Análise do Comportamento Aplicada em assuntos relacionados a saúde. Como apontado por Calheiros et al. (2016) e Marin et al. (2017), existem aspectos metodológicos que dificultam o acesso a esta intersecção entre a ciência analítica comportamental e a área de saúde, sendo um deles, a dificuldade para se encontrar termos ou palavras-chave abrangentes o suficiente para não restringir a busca em nenhuma das duas vertentes (Análise do Comportamento e Saúde).
Para tentar resolver possíveis problemas com busca de dados e inserção de artigos, tivemos como objetivo norteador desta revisão de literatura, identificar estudos, de uma revista específica da Análise do Comportamento Aplicada Uournal of Applied Behavior Analysis), publicados entre 2000 e 2015, que apresentassem contribuições à área de Saúde. Com base nisto, identificar e analisar os aspectos metodológicos utilizados e quais são os principais resultados de intervenções aplicadas no contexto de saúde.

\section{Método}

Como base de dados foi utilizada a plataforma online $\mathrm{JABA}^{1}$ que publica estudos originais envolvendo aplicação e análise de resultados em problemas de relevância social (Baer et al., 1968, 1987). O procedimento desta revisão foi dividido em três etapas, denominadas de: 1) Seleção, 2) Sistematização dos dados e 3) Análise de Resultados.

\section{Seleção}

Todos os volumes (com quatro edições por ano, do periódico), publicados nos últimos dezesseis anos foram utilizados para a revisão. Os artigos cujos títulos tivessem alguma relação com a área da saúde foram selecionados e visualizados integralmente.

Foi adotado como critério para seleção de estudos, a demonstração da relação entre alterações de padrões comportamentais e consideráveis melhorias para a saúde do sujeito, o que se configura como uma relação entre a Saúde e a Análise do Comportamento.

\section{Sistematização de dados}

Depois de selecionados os artigos relacionados a saúde nos 16 volumes publicados, entre 2000 e 2015, a etapa seguinte envolveu a leitura e análise dos dados produzidos e apresentados pelas publicações.

A sistematização de dados foi feita através de uma tabela elaborada no programa Microsoft Excel $2013{ }^{\circledR}$, na qual foram registradas as informações referentes aos temas investigados, métodos utilizados e resultados obtidos pelas intervenções. A análise dos artigos consistiu na formulação de classificações dos artigos selecionados, com base em suas semelhanças temáticas e, ou metodológicas.

\footnotetext{
${ }^{1}$ http://onlinelibrary.wiley.com/journal/10.1002/(ISSN)1938-3703).
} 


\section{Análise de resultados}

A partir dos dados inseridos na planilha, foram identificadas as intervenções e os resultados obtidos pelas intervenções apresentadas em cada artigo, analisando-se para cada tema as similaridades e disparidades de cada estudo.

\section{Resultados}

O Journal of Applied Behavior Analysis publicou nos 16 anos revisados (2000 a 2016) 1.242 artigos, disponíveis integralmente em sua plataforma on-line. Estes, estavam apresentados em 64 edições, publicadas trimestralmente, nos 16 volumes (vol. 33 a vol. 48). Deste montante de artigos, 160 (12,88\%) abordaram questões relacionadas a saúde e foram selecionados conforme os critérios anteriormente apresentados. Foi possível constatar, no período abarcado por esta revisão, que há uma média, de dez artigos por ano que abordam questões referentes à saúde. A Figura 1 apresenta os temas selecionados a partir da leitura e análise dos estudos selecionados.

No total foram encontrados 11 temas referentes a saúde. "Problemas Alimentares" foi o tema de saúde mais abordado pelos estudos, somando um total de 50 artigos, o que representou $31,25 \%$ da amostra. Foram considerados neste tema os estudos que analisaram e interviram sobre quaisquer problemas que estivessem relacionados aos hábitos alimentares de um determinado sujeito, como problemas de deglutição ou mastigação, alta seletividade de alimentos, packing ou expulsão de alimentos, ruminação, problemas intestinais, autonomia e até comportamentos concorrentes na hora de alimentar-se.

As intervenções, em sua maioria, realizaram procedimentos de reforçamento diferencial, aproximação sucessiva, bloqueio de fuga, controle de estímulos, reforçamento não contingente, além de esquemas de reforçamento para diminuição do custo de respostas para problemas de deglutição. Estas intervenções comportamentais aumentaram a frequência de comportamentos relacionados a hábitos saudáveis como, por exemplo: o aumento de variabilidade de alimentos consumidos, ou diminuição dos comportamentos que dificultam a alimentação. Quatro estudos também realizaram análises de contingências para compreender os controles que afetam comportamentos

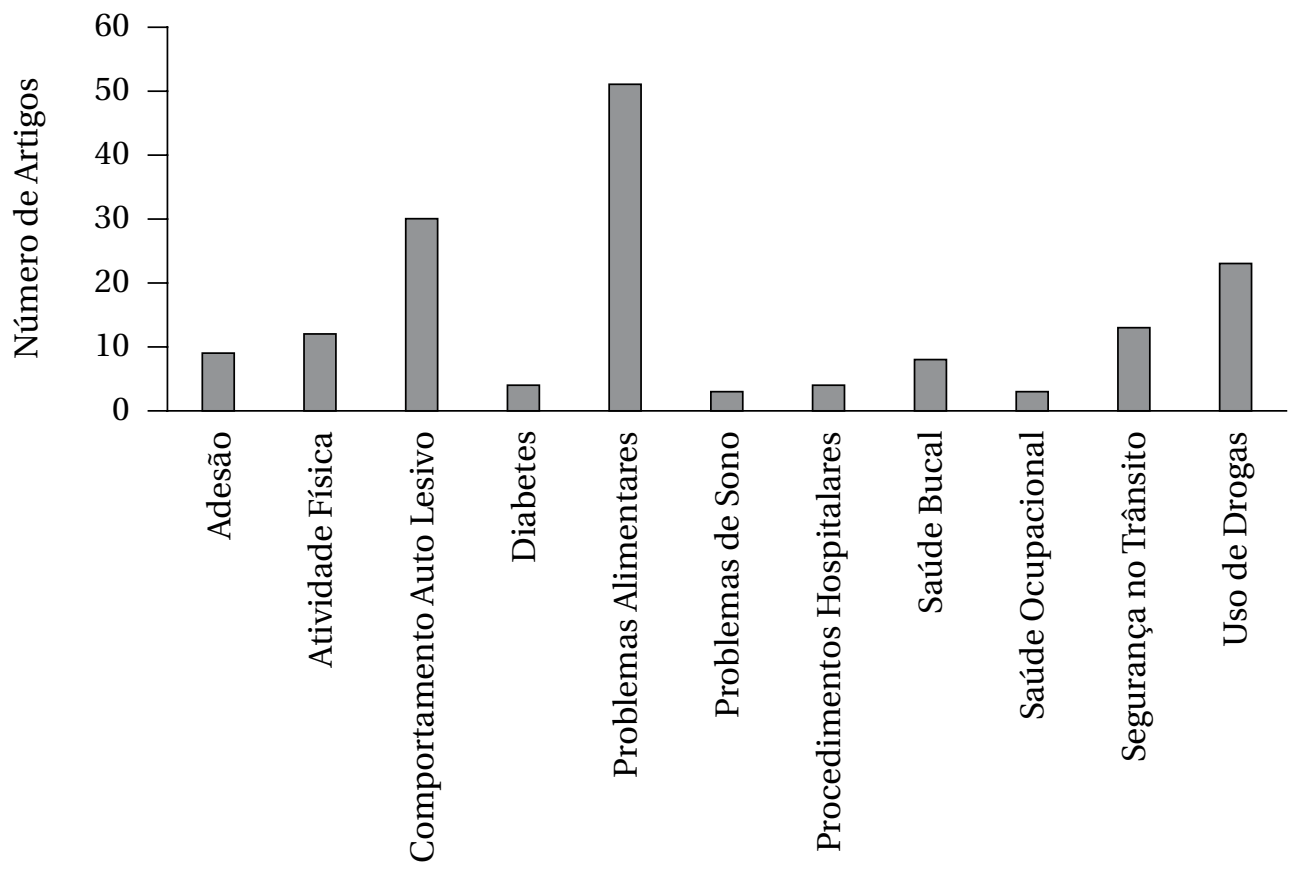

Temas em Saúde

Fonte: Elaborada pelos autores.

Figura 1

Número de artigos para cada tema de saúde encontrado pela revisão. 
que concorrem com práticas saudáveis. A utilização de instrumentos (colheres especiais, copos, mamadeira etc.) foi acompanhada de intervenções comportamentais, que visaram a redução de problemas como packing ou dificuldades da deglutição.

As intervenções comportamentais, portanto, mostraram-se eficazes para a análise de variáveis que interferem nas respostas de alimentar-se, além de possibilitar o manejo comportamental, produzindo condições favoráveis e dificultando a ocorrência de situações concorrentes. Além disto, três artigos também descrevem o treinamento de pais para auxiliar as intervenções relacionadas a problemas alimentares.

Ao todo foram encontrados 31 estudos (19,37\%) voltados ao tema "comportamento autolesivo" - comportamentos motores que produzem dano físico ou psicológico ao próprio sujeito, como beliscar-se, esmurrar-se, autocrítica demasiada ou, também, a ingestão de materiais não comestíveis. Nestes artigos, as intervenções visaram compreender as condições para a ocorrência de respostas autolesivos, de modo a promover a diminuição das mesmas.

Para a análise funcional dos casos de autoinjúria os pesquisadores manipularam variáveis, por meio de delineamentos de reversão e utilizaram reforçadores para a manutenção de outras respostas concorrentes. Os estudos apresentaram procedimentos de reforçamento diferencial (de comportamentos alternativos), bloqueio de resposta, retirada de reforçadores, análise de reforçadores sociais, reforçamento não contingentes e esquemas de reforçamento para redução de comportamentos alvo.

Os resultados dessas intervenções, mostraram-se eficazes no que diz respeito a análise de contingências que mantém comportamentos autolesivos. As intervenções também apontam soluções para a redução de comportamentos que produzem dano ao sujeito, além de apresentar várias utilidades dos princípios comportamentais que, quando combinados entre si, apresentam resultados promissores, como é o caso da aplicação conjunta de reforçamento diferencial de comportamentos alternativos e bloqueio de fuga (Jennet, Jann, \& Hagopian, 2011; Wilson et al., 2012).

Com referência ao "uso de drogas" foram encontrados 23 artigos (14,37\%). Este tema caracteriza-se por estudos que abordaram o controle ou o aumento do período de abstinência para uso de substâncias (álcool, maconha, cocaína etc.). As problemáticas abordadas dentro deste tema, apresentaram uma alta variabilidade no que diz respeito as intervenções e resultados alcançados.

Nos 23 artigos encontrados, nos procedimentos empregados, foi mais frequente o uso de: reforçamento positivo, reforçamento negativo, esquemas de reforçamento e também controle condicional para diminuição do consumo de drogas, ou para o aumento do período de abstinência. Os resultados mostraram a eficiência da utilização de técnicas de manejo comportamental para a obtenção de resultados positivos para o controle do comportamento de "usar drogas". A participação de sujeitos em sessões de terapia para tratamento do uso de drogas, com as situações que utilizaram procedimentos de manejo de contingências, apresentaram um maior, embora moderado, índice de participação dos usuários em relação a outros tipos de terapias (Ledgerwood, Alessi, Hanson, Godley, \& Petry, 2008). Neste mesmo sentido, Alessi, Petry e Urso (2008), mostraram que o uso de intervenções com manejo de contingências é mais eficaz para redução do consumo de cigarros, quando comparado ao tratamento padrão. Outra intervenção identificada foi a utilização de vouchers ou acompanhamento pela internet para manejo de contingências do comportamento de fumar (Dallery, Raiff,, \& Gabrinsk, 2013), para o qual além da intervenção, realizou-se também uma comparação entre reforçamento contingente (CR) e não contingente (NCR), apontando, assim como outros estudos para a maior eficiência do primeiro para redução do uso de drogas.

Foram considerados artigos de "segurança no trânsito" aqueles que interviram com hábitos de segurança que servem ao propósito da prevenção de danos. Neste tema, foram encontrados 13 estudos, representando $8,12 \%$ do total. Um problema muito citado nos estudos desse tema foi a tentativa de aumentar a frequência de respostas que representam hábitos de segurança, como por exemplo: o uso de cinto de segurança (Clayton, \& Helms, 2009), uso de capacete em ciclistas crianças (Van Houten, Van Houten, \& Malefant, 2007), adesão a sinais de trânsito (Austin, Hackett, Gravina, \& Lebbon, 2006; Cox, Cox \& Cox, 2000), entre outros.

Do total de estudos encontrados, seis deles abordaram o tema "uso do cinto de segurança", para alteração dos padrões de respostas dos sujeitos que fizeram uso de sinalizações visuais (cartazes, placas etc.) (Clayton \& Helms, 2009; Cox, Cox, \& Cox, 2000, 2005; Gras, Cunnill, Planes, Sullman, \& Oliveras 2003) 
e alterações mecânicas no carro, contingentes ao uso do cinto de segurança (Van Houten, Hilton, Schulman \& Reagan, 2011; Van Houten, Malefant, Reagan, Sifrit, Compton, \& Tenenbaum, 2010).

Outros estudos analisaram procedimentos de sinalização e reforçamento de comportamentos adequados, para problemas como utilização do celular enquanto indivíduos dirigem e utilização de capacete para andar de bicicleta. Uma outra intervenção foi a transmissão de informação, considerando sua consistência para a alteração do comportamento de ciclistas de usar capacete (Van Houten et al., 2007). Estes estudos, por sua vez, demonstraram um alto índice de resultados que alteraram os padrões de respostas no trânsito. Desta forma, pode-se considerar que os manejos comportamentais realizados, promoveram práticas mais seguras no trânsito, o que configura como uma atuação preventiva.

Os estudos que tiveram como objetivo principal o desenvolvimento de "Atividades físicas" somaram 12 artigos (7,5\%). O tema foi definido como intervenções que abordavam promoção e recuperação de estados saudáveis por meio de exercícios físicos. As intervenções, utilizaram reforçamento, em sete artigos, para aumentar, ou estabelecer repertórios de atividade física para a melhoria das condições de saúde.

Dois estudos (Fogel, Miltenberger, Graves, \& Koehler, 2010; Shayne, Fogel, Miltenberger, \& Koehler, 2012) analisaram o uso de "exergame" (jogos virtuais de esportes) em aulas de educação física, o que produziu um aumento de atividade física quando esta manipulação era inserida. No geral, os estudos que manejaram antecedentes ou consequentes analisaram principalmente os níveis de atividades físicas realizadas. Assim como para o tema "uso de drogas", um estudo utilizou a internet para manejo de contingências que promovam aumento ou manutenção de taxas de exercícios benéficos (Kurti ,\& Dallery, 2013).

Nove artigos foram definidos como "adesão" (5,62\%), nos quais foram avaliados os repertórios para seguimento de prescrições de comportamentos saudáveis. As intervenções variaram entre bloqueio de respostas concorrentes (disruptivas) e reforçamento de respostas de adesão, a qual teve como objetivo a aceitação e a participação ativa em procedimentos médicos. Todos os estudos analisados manejaram situações específicas de: uso de próteses, óculos, medicamentos, ou cooperação para execução de exames médicos ou terapias.
As intervenções utilizadas, sustentaram-se em procedimentos de aproximação sucessiva, bloqueio de fuga, fuga contingente e não contingente, reforçamento não contingente (NCR) e, também, esquemas de reforçamento. Os resultados demonstram que os procedimentos comportamentais aumentam repertórios cooperativos ou reduzem padrões de respostas disruptivas de sujeitos em tratamentos.

Como "saúde bucal" foram selecionados oito artigos $(5,0 \%)$ e envolveram intervenções de manejo voltados às práticas que prejudicam ou promovem a saúde oral de um sujeito. Os problemas analisados foram chupar o dedo, bruxismo, problemas vocais e comportamentos de cooperação em tratamentos odontológicos. Os dois estudos selecionados analisaram o comportamento de chupar o dedo, foram utilizados procedimentos de Awareness Enhacement Device (AED) para sinalizar ao sujeito quando esta resposta era emitida. Para os que interviram para o contexto do tratamento (consulta) odontológica, os procedimentos avaliados foram de reforçamento negativo não contingente e contingente. Os resultados demonstraram a redução ou o fortalecimento de repertórios que promovem condições melhores de saúde bucal.

O tema "diabetes" somou quatro artigos (2,5\%). Os estudos interviram no aumento da autopercepção, na redução do uso de testes glicêmicos e, para um deles (Shabani, \& Fisher, 2006), para a dessensibilização para medo de agulha. Em dois estudos os sujeitos foram treinados, por meios diferentes, checklist e redução de testes, para aumentar a percepção de taxas glicêmicas. Em Shabani e Fisher (2006), os experimentadores utilizaram dessensibilização sistemática para redução do medo de agulha, possibilitando, para o sujeito experimental, a realização de testes glicêmicos. Assim como em "atividade física" e "uso de drogas", um dos estudos também utilizou a internet para o manejo de contingências que favoreciam o aumento da autopercepção (Raiff, \& Dallery, 2010).

Os artigos sobre "Problemas Hospitalares" somaram também, quatro artigos (2,5\%). Para este tema, foram consideradas intervenções dirigidas às equipes de profissionais, avaliando a segurança dos pacientes e dos responsáveis pela equipe médica. Em todos estes artigos foi utilizado feedback para estabelecimento ou fortalecimento de repertórios de segurança para equipe hospitalar ou pacientes. As respostas foram lavar as mãos, posição para segurar o paciente, 
comunicação durante cirurgia e visita preventiva. Estas intervenções apresentaram resultados positivos para propagação de condições seguras para os sujeitos envolvidos com procedimentos hospitalares.

Em relação à "saúde ocupacional" foram encontrados três artigos $(1,87 \%)$. Estes avaliaram a qualidade da saúde de trabalhadores. Todos os três avaliaram o problema de posições durante o trabalho, o que pode ocasionar problemas posturais. Estes buscaram, também por meio de feedback, aprimorar as condições de autopercepção para questões posturais, fazendo com que funcionários discriminassem (Sigurdsson, Ring, Needham, Boscoe, \& Silverman, 2011) posturas corretas utilizando um computador. Os estudos aumentaram a autopercepção dos trabalhadores e, em um nível preventivo, melhoraram as condições do trabalho.

Por fim, com o tema "problemas do sono" foram encontrados três artigos (1,87\%). Como problemas do sono, foram considerados aqueles que analisaram situações referentes a problemas na hora de dormir, principalmente com crianças. As intervenções manipularam condições de atenção e interrupções de respostas para crianças com problemas no momento da hora de dormir. As intervenções realizadas diminuíram a frequência de comportamentos disruptivos que concorrem com a hora do sono, e envolveram alterações até mesmo de repertórios parentais, assim como em "Problemas de alimentação".

Dois tipos de delineamentos experimentais foram observados durante a análise: Delineamentos de grupo e sujeito como seu próprio controle (Andery, 2010; Cozby, 2006; Matos, 1999; Sampaio, Azevedo, Cardoso, Lima, Pereira, \& Andery, 2008). O delineamento de sujeito-único produz uma série de condições que servem para comparação entre os dados iniciais (linha de base) e finais (pós-intervenção) do próprio sujeito. Por outro lado, o delineamento entre grupos, produz dados em diversas condições para cada grupo, as quais são comparadas, entre os grupos, ou seja, entre as condições propostas estes. Do total, $78,75 \%$ ( $\mathrm{n}=126$ ) usaram o delineamento de sujeito único para analisar as intervenções, enquanto $21,25 \%$ dos estudos fizeram análise de grupos.

A Figura 2 apresenta os delineamentos experimentais (sujeito único e grupo) em relação aos temas voltados à saúde. Como mencionado anteriormente, "sujeito único" foi o agrupamento mais encontrado no geral, com exceção em dois temas ("Segurança no trânsito" e "Uso de drogas"). Para os temas "segurança no trânsito" e "uso de drogas" a maioria dos artigos realizou a comparação entre grupos para análise de resultados. No primeiro tema (segurança no trânsito), o único artigo publicado com delineamento de sujeito único abordou a questão do uso de capacete de proteção (para andar de bicicleta) com três crianças escolares (Van Houten et al., 2007).

Quando se analisa o número de participantes dos estudos, em relação aos delineamentos, nota-se que existe um número menor de participantes nos de estudos de "sujeito único" (1 a 10 participantes). Por outro lado, quando o número de participantes das pesquisas aumenta, o número de estudos com delineamento de sujeito único diminui e o de grupo torna-se mais frequente. Cabe salientar, portanto, que artigos de temas que frequentemente utilizam grupos para intervenções, possuem maior número de participantes. Por exemplo, para segurança no trânsito, o número de participantes em todos os estudos que utilizaram delineamento de grupo, tem um número de participantes acima de 100.

No geral, os temas encontrados apresentaram uma média constante de publicações, com exceção para "uso de drogas" que, no ano de 2008, apresentou uma edição exclusiva para o tema. Temas como "segurança no trânsito" e "atividade física" apresentaram variações conforme o tempo, no entanto, nada que possa ser caracterizado como um padrão mais ou menos sistemático nas publicações da revista.

\section{Discussão}

Os dados obtidos pela análise dos artigos encontrados no JABA, revelam a utilização das intervenções desenvolvidas pela ciência da Análise do Comportamento em contextos relacionados a saúde. Isto, por sua vez, pode representar uma atuação em múltiplas áreas, como uma ciência e uma tecnologia (Lattal, 2005).

As intervenções analisadas por esta revisão permitem considerar a saúde do sujeito de forma multideterminada e relacional, compreendendo como comportamentos e fatores que os controlam podem interferir nos estados de saúde de um indivíduo a partir de suas relações ambientais. (Engel, 1980; Reis, 1999; Straub, 2014; Taylor, 1999). Além disso, pode-se observar, nas intervenções e resultados descritos, a utilização clara de princípios comportamentais para fundamentação de intervenções que buscam a instauração ou modificação de repertórios através da seleção pelas consequências (Matos, 1999). 


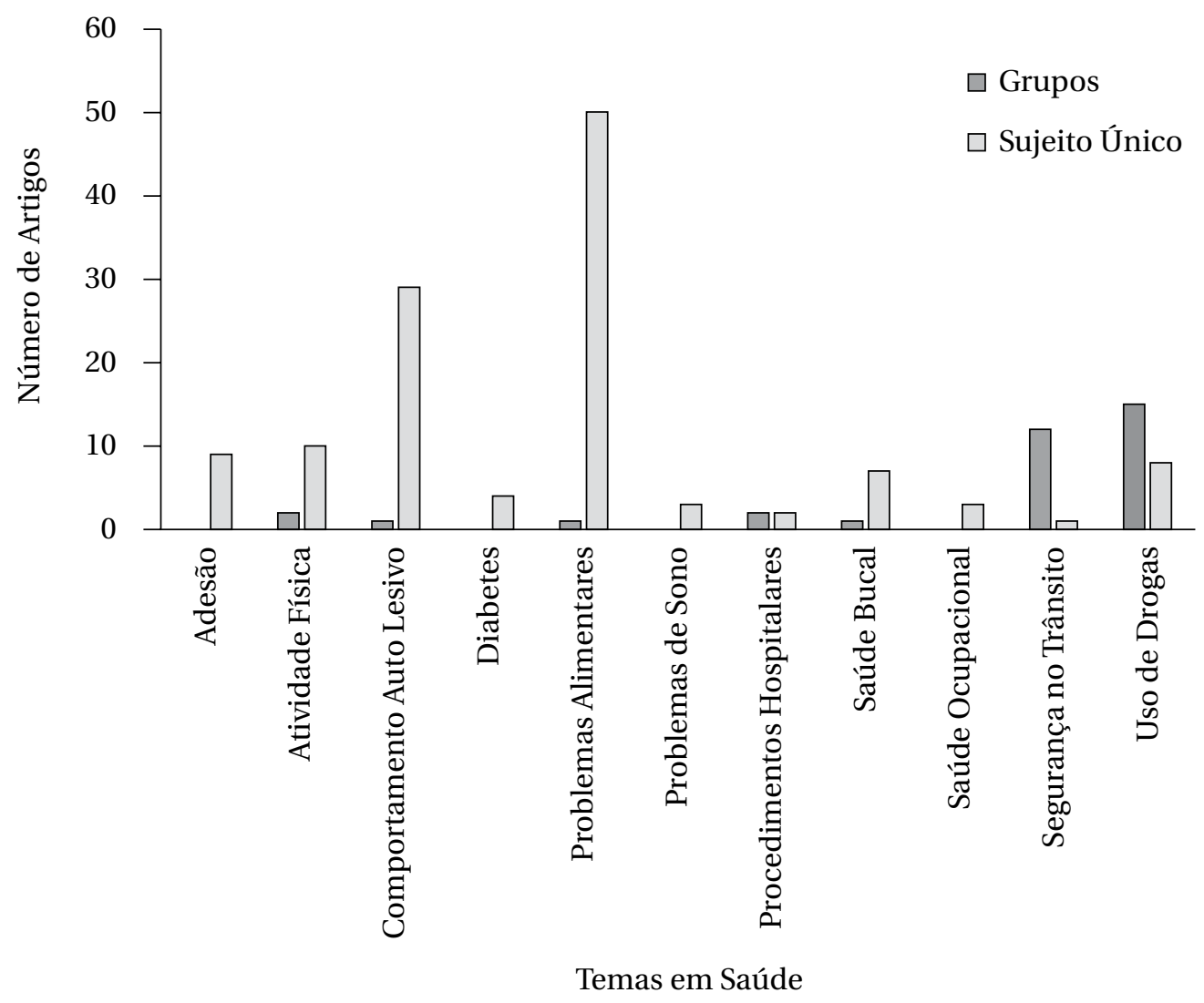

Fonte: Elaborada pelos autores.

Figura 2

Número dos delineamentos experimentais utilizados para cada tema de saúde.

Segundo Cooper, Heron e Heward (1987), a Análise do Comportamento Aplicada configura-se como uma ciência definida pelo seu foco em comportamentos que apresentam relevância social. As intervenções encontradas, por sua vez, podem melhorar as condições de saúde da sociedade, na relação entre os procedimentos utilizados. Baer et al. (1968) elaboraram sete dimensões chaves para considerar um estudo como comportamentalmente aplicado ("aplicado", "comportamental", "analítica", "tecnológico", "conceitualmente sistemático", "generalizável" e "efetivo").

Os artigos encontrados por esta revisão atendem aos critérios estabelecidos para uma Análise do Comportamento Aplicada, tendo sido demonstrado, principalmente, como as sustentações teóricas básicas ("conceitualmente sistemática"), utilizadas nas intervenções, produzem resultados satisfatórios ("efetivos") e replicáveis entre os estudos, o que se denota pelas citações de estudos anteriores, publicados também no periódico ("generalizável"). Sobre esta questão, foi possível observar que, assim como apontado por Elliot, Morgan, Fuqua, Ehrhardt e Poling (2005), os estudos publicados no JABA tem um alto índice de citação interna, o que pode representar uma continuidade no desenvolvimento de pesquisas deste periódico.

Em consideração aos temas, foi possível observar, assim como em outras revisões (Marin et al., 2017; Moraes, \& Rolim, 2013), uma variedade em que a Análise do Comportamento produz conhecimento e técnicas aplicáveis (Lattal, 2005). Neste sentido, como apontado por Calheiros (2016), a maioria dos estudos analisados utilizou delineamento de sujeito único para as intervenções, uma característica comum em estudos da Análise do Comportamento. Isto, reflete-se em uma grande parcela dos estudos com um baixo número de sujeitos experimentais. Dados referentes as intervenções e delineamentos experimentais podem estar correlacionados com características dos temas, objetivos, comportamentos alvos e também as próprias características dos periódicos, como é o caso do JABA. Em consequência disto, a revisão de um 
único periódico pode representar uma fraqueza para compreensão das múltiplas intervenções e metodologias que são utilizadas pela Análise do Comportamento em múltiplos contextos.

\section{Considerações Finais}

A revisão, em seu total, possibilita a avaliação das intervenções e manejos realizados pelos expe- rimentos, os quais, por sua vez, apresentam resultados positivos na alteração dos comportamentos e promoção de estados mais saudáveis. Por sua vez, a análise dos temas demonstra a utilização de pressupostos de uma ciência aplicada, baseada em uma ciência básica, que propicia o desenvolvimento de intervenções generalizáveis para outros contextos (Baer et al. 1968, 1987; Lattal, 2005).

\section{Referências}

Alessi, S. M., Petry, N. M., \& Urso, J. (2008). Contingency management promotes smoking reductions in residential substance abuse patients. Journal of Applied Behavior Analysis, 41(4), 617-622. https://doi.org/10.1901/ jaba.2008.41-617

Andery, M. A. P. A. (2010). Métodos de pesquisa em análise do comportamento. Psicologia USP, 21(2), 313-342. https://doi.org/10.1590/S0103-65642010000200006

Austin, J., Hackett, S., Gravina, N., \& Lebbon, A. (2006). The effects of prompting and feedback on drivers'stopping at stop signs. Journal of Applied Behavior Analysis, 39(1), 117-121. https://doi.org/10.1901/jaba.2006.49-04

Baer, D. M., Wolf, M. M., \& Risley, T. R. (1968). Some current dimensions of applied behavior analysis. Journal of Applied Behavior Analysis, 1(1), 91-97. https://doi.org/10.1901/jaba.1968.1-91

Baer, D. M., Wolf, M. M., \& Risley, T. R. (1987). Some still-current dimensions of applied behavior analysis. Journal of Applied Behavior Analysis, 20(4), 313-327. https://doi.org/10.1901/jaba.1987.20-313

Banaco, R. A. (1999). Técnicas cognitivo-comportamentais e análise funcional. In R. R. Kerbauy (Org.), Sobre comportamento e cognição: Psicologia comportamental e cognitiva: Da reflexão teórica à diversidade de aplicação (v. 4, pp. 75-82). Santo André: Esetec.

Calheiros, T. C. (2016). Análise do comportamento aplicada às doenças crônicas não transmissíveis: Uma revisão sistemática da produção brasileira stricto sensu (Dissertação de mestrado). Universidade Estadual de Londrina, Londrina, PR, Brasil.

Calheiros, T. C., Amaral, M., Gon, M. C. C., \& Costa Júnior, Á. L. (2016). Análise do comportamento e saúde: levantamento e análise de artigos em bases de acesso livre. Temas em Psicologia, 24(3), 947-961. https://doi.org/10.9788/ TP2016.3-10Pt

Clayton, M. C., \& Helms, B. P. (2009). Increasing seat belt use on a college campus: An evaluation of two prompting procedures. Journal of Applied Behavior Analysis, 42(1), 161-164. https://doi.org/10.1901/jaba.2009.42-161

Cooper, J. O., Heron, T. E., \& Heward, W. L. (1987). Applied behavior analysis. Princeton: Marril.

Cox, B. S., Cox, A. B., \& Cox, D. J. (2000). Motivating signage prompts safety belt use among drivers exiting senior communities. Journal of Applied Behavior Analysis, 33(4), 635-638. https://doi.org/10.1901/jaba.2000.33-635

Cox, C. D., Cox, B. S., \& Cox, D. J. (2005). Long-term benefits of prompts to use safety belts among drivers exiting senior communities. Journal of Applied Behavior Analysis, 38(4), 533-536. https://doi.org/10.1901/jaba.2005.34-03

Cozby, P. C. (2006). Métodos de pesquisa em ciências do comportamento. São Paulo: Atlas.

Dallery, J., Raiff, B. R., \& Grabinski, M. J. (2013). Internet-based contingency management to promote smoking cessation: A randomized controlled study. Journal of Applied Behavior Analysis, 46(4), 750-764. https://doi.org/10.1002/jaba.89

Elliot, A. J., Morgan, K., Fuqua, R. W., Ehrhardt, K., \& Poling, A. (2005). Self-and cross-citations in the Journal of Applied Behavior Analysis and the Journal of Experimental Analysis of Behavior: 1983-1992. Journal of Applied Behavior Analysis, 27(4), 729-731. https://doi.org/10.1901/jaba.1994.27-729

Engel, G. L. (1980). The clinical application of the biopsychosocial model. American Journal of Psychiatry, 137(5), 535-544. https://doi.org/10.1176/ajp.137.5.535 
Fogel, V. A., Miltenberger, R. G., Graves, R., \& Koehler, S. (2010). The effects of exergaming on physical activity among inactive children in a physical education classroom. Journal of Applied Behavior Analysis, 43(4), 591-600. https:// doi.org/10.1901/jaba.2010.43-591

Gon, M. C. C., Zakir, N. S., Andrade, L. V., Gavazzoni, A., \& Brandenburg, O. J. (2015). Possíveis relações entre comportamento e condições de saúde. In V. Bender, \& S. R. Souza, Análise do comportamento aplicada a diferentes contextos (pp.75-94). Londrina: EDUEL.

Gorayeb, R. (2010). Psicologia da saúde no Brasil. Psicologia: Teoria e Pesquisa, 26(n. spe), 115-122. https://doi. org/10.1590/S0102-37722010000500010

Gorayeb, R., \& Guerrelhas, F. (2003). Sistematização da prática psicológica em ambientes médicos. Revista Brasileira de Terapia Comportamental e Cognitiva, 5(1), 11-19. Recuperado de http://pepsic.bvsalud.org/scielo. php?script=sci_arttext\&pid=S1517-55452003000100003

Gras, M. E., Cunill, M., Planes, M., Sullman, M. J., \& Oliveras, C. (2003). Increasing safety-belt use in Spanish drivers: A field test of personal prompts. Journal of Applied Behavior Analysis, 36(2), 249-251. https://doi. org/10.1901/jaba.2003.36-249

Jennett, H., Jann, K., \& Hagopian, L. P. (2011). Evaluation of response blocking and re-presentation in a competing stimulus assessment. Journal of Applied Behavior Analysis, 44(4), 925-929. https://doi.org/10.1901/ jaba.2011.44-925

Kerbauy, R. R. (2002). Comportamento e saúde: Doenças e desafios. Psicologia USP, 13(1), 11-28. https://doi. org/10.1590/S0103-65642002000100002

Kubo, O. M., \& Botomé, S. P. (2001). Formação e atuação do psicólogo para o tratamento em saúde e em organizações de atendimento à saúde. Interação em Psicologia, 5(1). https://doi.org/10.5380/psi.v5il.3319

Kurti, A. N., \& Dallery, J. (2013). Internet-based contingency management increases walking in sedentary adults. Journal of Applied Behavior Analysis, 46(3), 568-581. https://doi.org/10.1002/jaba.58

Lattal, K. A. (2005). Ciência, tecnologia e análise do comportamento. In J. Abreu-Rodrigues, \& M. R. Ribeiro (Orgs.), Análise do comportamento: Pesquisa, Teoria e Aplicação (pp. 15-26). Porto Alegre: Artmed.

Ledgerwood, D. M., Alessi, S. M., Hanson, T., Godley, M. D., \& Petry, N. M. (2008). Contingency management for attendance to group substance abuse treatment administered by clinicians in community clinics. Journal of Applied Behavior Analysis, 41(4), 517-526. https:// doi.org/10.1901/jaba.2008.41-517

Marin, R., Moraes, A.B.A., \& Faleiros, P. B. (2017). Uma revisão de temas da saúde estudados pela análise do comportamento. In D. L. O. Vilas Boas, F. Cassas, H. L. Gusso, \& P. C. M. Mayer (Orgs.), Comportamento em foco (v. 6, pp. 156-168). São Paulo: Associação Brasileira de Psicologia e Medicina Comportamental.

Matarazzo, J. D. (1982). Behavioral health's challenge to academic, scientific, and professional psychology. American Psychologist, 37(1), 1. https://doi.org/10.1037//0003-066x.37.1.1

Matos, M. A. (1999). Análise funcional do comportamento. Estudos de Psicologia(Campinas), 16(3), 8-18. https://doi.org/10.1590/S0103-166X1999000300002

Mechanic, D. (1962). The concept of illness behavior. Journal of Chronic Diseases, 15(2), 189-194. https://doi. org/10.1016/0021-9681(62)90068-1

Moraes, A. B. A., \& Rolim, G. S. (2013). Contribuição da análise do comportamento para a psicologia da saúde. In Anais da Reunião Anual da Sociedade Brasileira de Psicologia, Ribeirão Preto, SP, Brasil, 43. Recuperado de http://www.r2013.inscricoes.sbponline.org.br/conteudo/view?ID_CONTEUDO=214

Raiff, B. R., \& Dallery, J. (2010). Internet-based contingency management to improve adherence with blood glucose testing recommendations for teens with type 1 diabetes. Journal of Applied Behavior Analysis, 43(3), 487-491. https:// doi.org/10.1901/jaba.2010.43-487

Reis, J. (1999). Modelo metateórico da Psicologia da Saúde para o séc. XXI: Interação ou integração biopsicossocial? Análise Psicológica, 17(3), 415-433.

Sampaio, A. A. S., Azevedo, F. H. B. D., Cardoso, L. R. D., Lima, C. D., Pereira, M. B. R., \& Andery, M. A. P. A. (2008). Uma introdução aos delineamentos experimentais de sujeito único. Interação em Psicologia, 12(1), 151-164. https://doi.org/10.5380/psi.v12il.9537 
Shabani, D. B., \& Fisher, W. W. (2006). Stimulus fading and differential reinforcement for the treatment of needle phobia in a youth with autism. Journal of Applied Behavior Analysis, 39(4), 449-452. https://doi.org/10.1901/ jaba.2006.30-05

Shayne, R. K., Fogel, V. A., Miltenberger, R. G., \& Koehler, S. (2012). The effects of exergaming on physical activity in a third-grade physical education class. Journal of Applied Behavior Analysis, 45(1), $211-215$. https://doi.org/10.1901/jaba.2012.45-211

Sigurdsson, S. O., Ring, B. M., Needham, M., Boscoe, J. H., \& Silverman, K. (2011). Generalization of posture training to computer workstations in an applied setting. Journal of Applied Behavior Analysis, 44(1), 157-161. https://doi. org/10.1901/jaba.2011.44-157

Skinner, B. F. (1991) Questões recentes na análise comportamental. Campinas: Papirus.

Starling, R. R. (2001). Análise funcional da enfermidade: Um quadro conceitual analítico-comportamental para orientar a intervenção psicológica em contextos médicos. In H. Guilhardi (Org.), Sobre comportamento e cognição (v. 8, pp. 262-296). Santo André: Arbytes.

Straub, R. O. (2014). Psicologia da saúde: Uma abordagem biopsicossocial (3a ed.). Porto Alegre: Artmed,. 528p.

Taylor, S. E. (1990). Health psychology: The science and the field. American Psychologist, 45(1), 40-50. https://doi. org/10.1037//0003-066x.45.1.40

Taylor, S. E. (1999). Health psychology (4a ed.). Boston: Mc Graw Hill.

Teixeira, J. A. C. (2004). Psicologia da saúde. Análise Psicológica, 22(3), 441-448. Recuperado de http://www.scielo. mec.pt/scielo.php?script=sci_arttext\&pid=S0870-82312004000300002

Van Houten, R., Hilton, B., Schulman, R., \& Reagan, I. (2011). Using accelerator pedal force to increase seat belt use of service vehicle drivers. Journal of Applied Behavior Analysis, 44(1), 41-49. https://doi.org/10.1901/ jaba.2011.44-41

Van Houten, R., Malenfant, J. E., Reagan, I., Sifrit, K., Compton, R., \& Tenenbaum, J. (2010). Increasing seat belt use in service vehicle drivers with a gearshift delay. Journal of Applied Behavior Analysis, 43(3), 369-380. https://doi.org/10.1901/jaba.2010.43-369

Van Houten, R., Van Houten, J., \& Malenfant, J. E. (2007). Impact of a comprehensive safety program on bicycle helmet use among middle-school children. Journal of Applied Behavior Analysis, 40(2), 239-247. https://doi. org/10.1901/jaba.2007.62-06

Wilson, D. M., Iwata, B. A., \& Bloom, S. E. (2012). Computer-assisted measurement of wound size associated with self-injurious behavior. Journal of Applied Behavior Analysis, 45(4), 797-808. https://doi.org/10.1901/ jaba.2012.45-797

\section{Ramon Marin}

Doutorando em Psicologia da Universidade Federal de São Carlos, São Carlos - SP. Brasil.

E-mail: rm.ramonmarin@gmail.com

(iD https://orcid.org/0000-0003-1099-3037

\section{Pedro Bordini Faleiros}

Professor Doutor da Universidade Metodista de Piracicaba (Unimep), Piracicaba - SP. Brasil.

E-mail: pbfaleiros@gmail.com

(iD https://orcid.org/0000-0003-3368-1415

Antonio Bento Alves de Moraes

Professor Colaborador da Faculdade de Odontologia de Piracicaba/Universidade de Campinas (Unicamp), Piracicaba - SP. Brasil.

E-mail: abento@unicamp.br

iD https://orcid.org/0000-0003-1432-5538 
Endereço para envio de correspondência:

Rua das Margaridas, 205, apto. 13. CEP: 13.566-543. São Carlos - SP. Brasil.

Recebido 04/05/2018

Reformulado 13/09/2018

Aceito 12/02/2019

Received 05/04/2018

Reformulated $09 / 13 / 2018$

Approved 02/12/2019

Recibido 04/05/2018

Reformulado 13/09/2018

Aceptado 12/02/2019

Este projeto contou com o financiamento da FAPESP (Projeto No 2014/26253-5) e com o apoio da Coordenação de Aperfeiçoamento de Pessoal de Nível Superior - Brasil (CAPES) - Código de Financiamento 001

Como citar: Marin, R., Faleiros, P. B., \& Moraes, A. B. A. (2020). Publicações em saúde pela análise do comportamento. Psicologia: Ciência e Profissão, 40, 1-13.. https://doi.org/10.1590/1982-3703003197787

How to cite: Marin, R., Faleiros, P. B., \& Moraes, A. B. A. (2020). Publicações em saúde pela análise do comportamento. Psicologia: Ciência e Profissão, 40, 1-13.. https://doi.org/10.1590/1982-3703003197787

Cómo citar: Marin, R., Faleiros, P. B., \& Moraes, A. B. A. (2020). Publicações em saúde pela análise do comportamento. Psicologia: Ciência e Profissão, 40, 1-13.. https://doi.org/10.1590/1982-3703003197787 